\title{
HYBRID ELECTRONIC HEALTH RECORDS
}

\author{
Tiago Pedrosa, Rui Pedro Lopes \\ Polytechnic Institute of Bragança, Portugal \\ pedrosa@ipb.pt,rlopes@ipb.pt \\ João C Santos, \\ Coimbra Institute of Engineering, DEE, Portugal \\ jcandido@isec.pt \\ Carlos Costa, José Luís Oliveira \\ University of Aveiro - IEETA, Portugal \\ carlos.costa@ua.pt, jlo@ua.pt
}

Keywords: $\quad$ EHR, PHR, Mobility, Hybrid EHR

\begin{abstract}
The research related with digital health records is has been a hot topic since the last two decades, producing diverse results namely two main types - Electronic Health Records and Personal Health Records. Based on those types of records different implementations appeared, although new challenges also emerged. The wider citizen mobility, liberalization of health care providing, alternative medicine, elderly care and remote patient monitoring are examples of the new types of challenges that the records have to cope with. Those new challenges brought more actors to the scene that can belong to different healthcare networks, private or public sector even from different countries. For creating a true longitudinal patient-centric electronic health record, those actors need to collaborate in the creation and maintenance of the record.

During the search of a solution for enabling the collaborative use and creation of the entire healthcare providing actors, EHR and PHR where studied to understand how they cope with the identified challenges and a requirement analysis was also performed. It is presented the Hybrid Electronic Health Record (HEHR) as a solution, describing how information can be created and used referring also how the patient defines the access control. It is also discussed new services that can make use of the HEHR.
\end{abstract}

\section{INTRODUCTION}

Digital health records had been on study on the last two decades, most of the deployed where based in two type of records - Electronic Health Records (EHRs) and Personal Health Records (PHRs).

The EHRs where defined and deployed mainly to cope with the requirements of the healthcare providers without considering patient needs on the process. On the other hand the PHRs where created to enable a more active role by the patient in the creation and maintenance of his health record. This two type of records, have huge differences that will be studied detailed ahead.

The idea of achieving a longitudinal patientcentered record that can enable health professionals to have an integrated view of the patient's clinical history, enabling the provision of better healthcare (Smith and Kalra, 2008) is still an open challenge, because the requirements, of a solution that can maintaining this type of record, have changed along time.

As technology evolved, substantial changes in healthcare provisioning and on the citizens' way of living had occurred. The liberalization of the healthcare providing brought more actors to the healthcare scene as well as new type of procedures that external providers offer to the patient (Chanda, 2002). Leading the patient to make a free choice of health provider, to access to new types of complementary and alternative medicine, among others.

The citizens' mobility had increased a lot, nowadays citizens travel, either for professional, personal or medical reasons (The European Economic and Social Committee and the comittee of regions, 2007). Hence, the citizens may change 
of residence during his lifetime, leading to a huge number of different healthcare providers, public or private one, federated or isolated, as well from different countries.

Along with these changes, the patient also urges to have more active role, controlling the access to his medical information, contributing to his record without compromising the choice of his healthcare provider. Hence, new approaches to the management of healthcare data, and new services that can make use of the patient health care information have appeared, requiring a format that could cope with this kind of paradigm (Eysenbach, 2008).

Along with all this changes in society, healthcare provisioning and also citizens' posture have a direct impact in the requirements of the digital health records. For achieving a longitudinal record all the new actors should collaborate in the creation, maintenance and use of the information on the records. The two main types of records, EHRs and PHRs, will be studied concerning the collaborative use of the records. The requirements of a record that cope with the collaboration of the actors are present in order to sustain the proposed solution a Hybrid Electronic Health Record. It is described how information can be created and used, referring also how the patient defines the access control, new services that can make use of the HEHR are also discussed.

\section{EHR VS PHR}

A analysis of the two main streams of records, EHRs and PHRs, is made to understand how the new challenges fit on them. The analysis is mostly oriented how and who can contribute to the record, the responsible of access control, level of sharing and who provides the systems that manipulate the records.

The Electronic Health Records (EHR) can be described as a longitudinal storage of patient health information generated by one or more encounters in any care delivery setting (HIMSS, 2010a). This information may include several kinds of data such as patient demographics, progress notes, problems, medications, vital signs, past medical history, immunizations, laboratory data, and radiology reports. The EHR has the ability to generate a complete record of a clinical patient encounter, as well as supporting other care-related activities directly or indirectly via external interfaces.
The information on EHRs is produced by healthcare professionals and maintained inside the healthcare providers. EHRs are deployed mainly in four types of models: the fully federated, federated, service orientated and integrated (NCRR, 2006). Also each deployment in each country/region or federation uses diverse approaches under different regulatory frameworks, plus the lack of a well defined standard makes even more difficult the interoperability (The Lancet, 2008), raising the challenges of free collaboration of all the actors. The deployment of EHRs is, typically, performed in a single institution or network, requiring agreements between the healthcare providers for interoperability and sharing. The need of such agreements hinders the use of electronic health record in a wider concept of mobility and freedom of choice for healthcare providers (between public/private actors and even from different countries).

Those systems are mainly devoted to facilitate the work and information flow between different departments of an institution or a federation. They allow the flow of information between diverse actors, i.e. specialist departments, radiology, or laboratory departments. They also, try to manage administrative information related to admission, discharge and payments (The Lancet, 2008). This approach excludes any intervention by the patient in every step, including the requirements analysis. In other words, it is a solution to cope with healthcare professionals needs, inside a well-defined group of actors, supported by agreements between them, to share patient clinical related information. Patient starts to be enabled to access some of the information, but he doesn't control it.

The Personal Health Records (PHR) can be described as an lifelong tool for managing relevant health information of an individual (HIMSS, $2010 \mathrm{~b})$. It promotes personal health information maintenance and may be used in a broader scope (entire health data) or in more specific scenarios, such as chronic disease management. The PHR is owned, managed, and shared by the individual or a legal proxy(s) and must be secure to protect the privacy and confidentiality of the health information it contains.

Although different types of PHR have been developed the most relevant are: 1) the standalone, resident in some external store device (Santos et al., 2010), and 2) the web-based, allowing patients to keep record of their personal medical data. The most prominent web-based PHR are 
Google Health, Microsoft HealthVault and Dossia. These web-based PHRs are generally based on a central repository and on a set of core features that, in some cases, can be extended by external third-party services. However, the use of external services need approval by the providers of the web-based PHRs.

Table 1 resumes the main differences between EHRs and PHRs. According to the definitions and the method of deployment of those types of records, the PHR seams to better cope with most of the needs, as it enables the easily sharing between different actors despite of their location, agreements and depends on patient approval. It also solves the problem of the infrastructure cost, as the patient chooses a PHR provider. It also empowers the patient to maintain and control the access to his medical record. One drawback is the trust by the clinical staff on the integrity of the clinical information, since the patient can update and create data without any kind of validation or mediation by the medical staff.

Table 1: EHR vs PHR

\begin{tabular}{|l|l|l|}
\hline & EHR & PHR \\
\hline Guardian & Providers & $\begin{array}{l}\text { Patient or a ser- } \\
\text { vice in his be- } \\
\text { half }\end{array}$ \\
\hline Creation of data & Medical Staff & $\begin{array}{l}\text { Patient or ex- } \\
\text { ported by ser- } \\
\text { vices }\end{array}$ \\
\hline Sharing & $\begin{array}{l}\text { Institutional } \\
\text { agreements }\end{array}$ & Patient choice \\
\hline Access Control & By the Provider & $\begin{array}{l}\text { Controlled by } \\
\text { the patient }\end{array}$ \\
\hline System Provider & Providers & $\begin{array}{l}\text { External Service } \\
\text { Provider }\end{array}$ \\
\hline
\end{tabular}

The EHR has the trust of the medical staff. However, a record sharing is a very hard procedure, only made in well-defined federations. It also makes difficult to enable the free choice of the patient's provider, since he is dependent on the agreements that providers have in other to access his medical information for provide proper healthcare. Hence, in a broader concept of mobility, the different regulatory frameworks raise the difficult of sharing medical records data between foreign providers. In this scenario, the patient is a passive actor, since he cannot contribute to his record, and also can't control the access to his medical information.

There are some models to overcome the challenges of the disperse data on EHR and also in medical imaging (Costa et al., 2009). In the case of the medical imaging solutions, a solution that enables indexing and retrieving DICOM data in disperse and unstructured archives, with this solution is possible to google like query for an exams and retrieve them despite of their location, as long their are in the same sharing federation.

For coping the mobility and EHRs exits a model that implements the patient intent consent to enable the sharing of sensible information between different healthcare actors. It promotes the transparent use of existent EHR systems in the healthcare providers, and when the providers doesn't have EHR systems, it provides a web-based PHR solution to save the new information (Pedrosa et al., 2010). This solution is based in the principle that all healthcare providers will open their systems to remote retrieving the patient information inside their EHR systems, the system will create a unified version of the disperse data on execution time. A better solution should enable that the record is in a common format and outside the providers EHR systems with complete access control by the patient, enabling easy collaboration and use of the information on the record.

\section{A NEW PROPOSAL FOR A HYBRID EHR}

The Hybrid Electronic Health Record appears as a solution to overcome the problems identified previously, namely enable the free collaboration of all the actors in healthcare, controlled by the patient and with medical data integrity control. The characteristics between EHRs and PHRs are complementary, so this hybrid approach tries to combine the two for achieving a solution where all actors involved in the healthcare can contribute and make use of the electronic health record. The patient himself will manage the access to his record, so the access to the infrastructure is regulated by the patient and will not depend on agreements between the healthcare providers.

For enabling the HEHR all actors are required to generate a report that will be considered as their contribution to the EHR. Those contributions can be generated from the systems already deployed, from user input or by specialized services. The aggregation of all contributions results in the patient-centric longitudinal electronic health record.

The HEHR is based in a centralized repository, trusted by the patient, to deposit all the contributions. The collaboration of all the actors 
is illustrated on Figure 1.

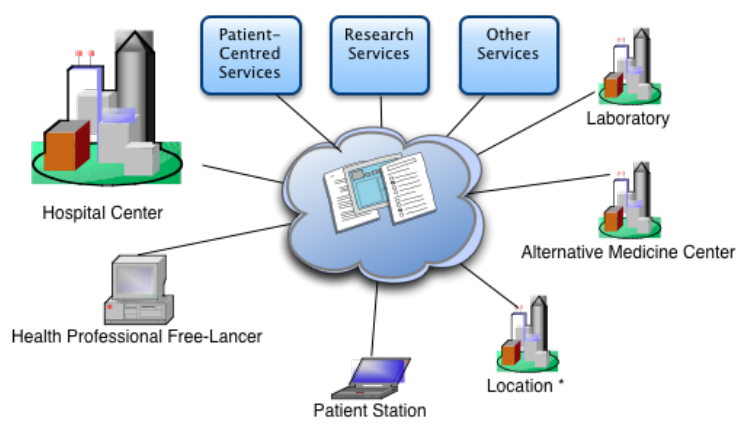

Figure 1: HEHR actors

The access control is performed through the patient station, where he can also create contributions to his record. Healthcare providers such as hospital centers, laboratories and other medical centers, can contribute by exporting reports from their systems or by using external services. All actors may contribute to the HEHR, when previously authorized by the patient. New services can manipulate the information as patient centeredservices, e.g. prescription alarms or other treatment alarms; research services that can be used by researchers when allowed by the patient to use his information for research proposes; and other new services that can bring added value to the use of patient clinic information.

The patient will control who can access his medical information and how. At the same time he can easily give access to actors in the healthcare or similar area, enabling the deployment of new services that can make use of his information. Each new producer or consumer that wants to gain access to the patient EHR requires the patient authorization. The clinical integrity of the contributions can be confirmed, increasing the trust on the system by the healthcare professionals.

\subsection{Use Cases}

On this type of record, three types of operation should be explained: the deposit of a contribution, the request of the record and how a new service can make use of the HEHR.

The operation of deposit of information can be decomposed in three steps, as illustrated on Figure 2: first a report in a standard format, such as CDA (Dolin et al., 2006), CCR (Ferranti et al., 2006) or OpenEHR archetypes (OpenEHR, 2007), in an XML serialized form, is generated. Then those reports have to be signed by the producers to ensure integrity and traceability. The last step is the deposit of the information on the repository chosen by the patient, if the requester has enough privileges.

For scenarios that already have EHR systems in use, those systems should be able to generate that reports, sign and deposit in a seamless way. As an alternative, a local service should do those steps on the healthcare provider. If the provider doesn't have an already deployed system, he can create or choose a service provider that enable him create the contribution.

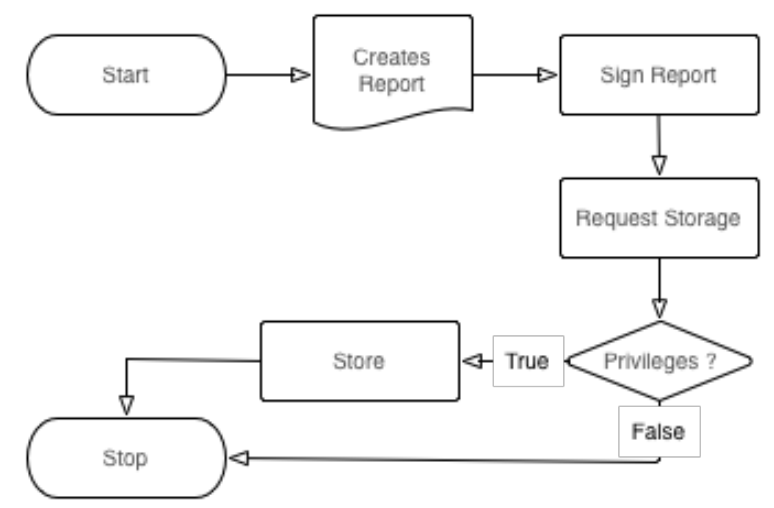

Figure 2: HEHR store procedure

The retrieval process is explained in Figure 3. The actor can request the full record or parts of it. The system will check the requester privileges, and will see if the requester wants the contributions individually or assembled as a unique view. The policies previously defined by the patient is applied on the contributions set in order to create a particular view for the user.

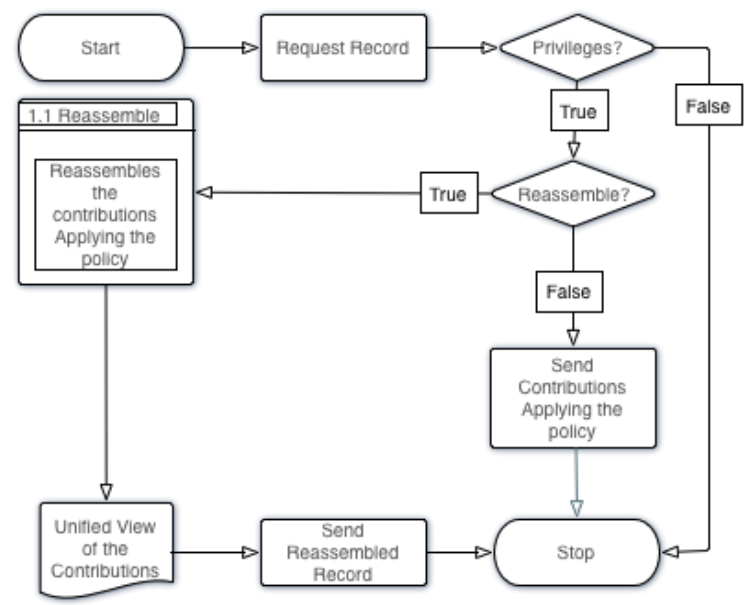

Figure 3: HEHR retrieve procedure

The possibility of asking for the contributions before unification, allows creating custom views of the data, associated with a navigation model. 
Other important aspect in the HEHR is how an actor can make use of the information, the process is illustrated on the Figure 4.

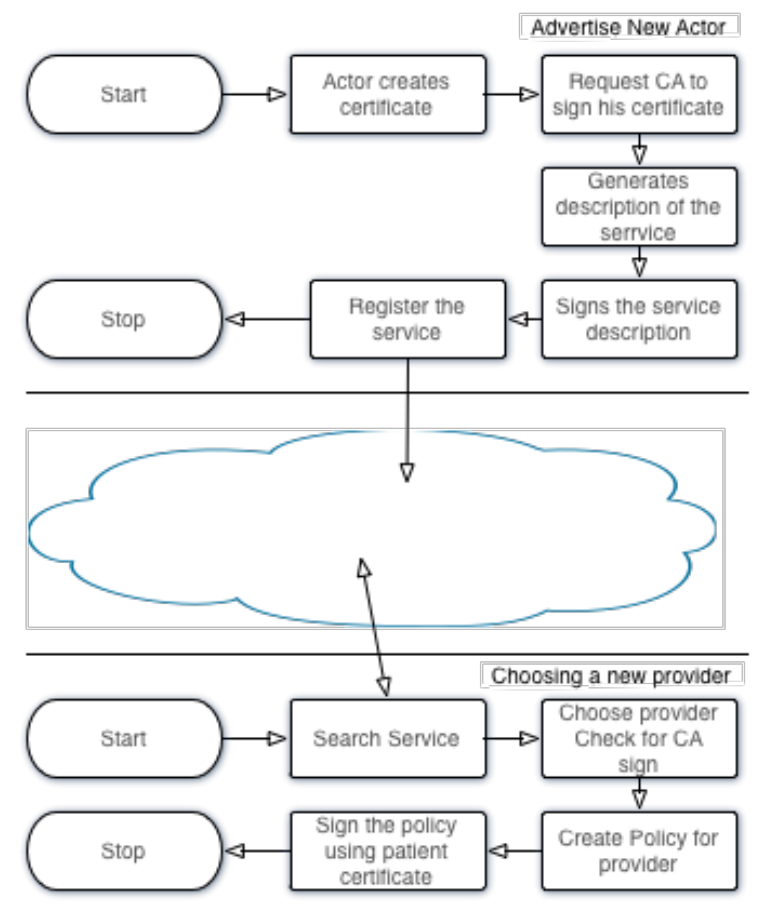

Figure 4: HEHR joining new actor

The process is defined in two main sub processes: the advertisement of a new actor providing a service and the procedure of a patient choosing a new service provider. In the former, a new service should generate a certificate and ask a Certification Authority (CA) to sign it. Then, the actor should create a description of the service, sign it, and register it. The actors can be services or healthcare professionals that want to deposit or make use of the information on the record.

When a patient wants to use a new service, he searches the service, chooses the provider and validates the CA signature. Then, using the public certificate of the chosen service, the patient defines the policy for that actor, controlling what the service/actor can view or store in his record.

\section{DISCUSSION}

The HEHR tries to create a true longitudinal patient-centric electronic health record, based on contributions from all actors that provides healthcare services to the patient. This open collaboration, only controlled by the policies specified by the patient, can deal with the mobility and freedom of choice of the patient, since all they can easily join as patient collaborators. The bureaucratic sharing problem between actors is solved by the use of the patient consent.

It is also brought to the healthcare professionals the trust in the clinical integrity, since its signature can check all collaborations integrity, so practitioners can use it with similar confidence of records on an EHR system.

Considering the features of the EHR and PHR, described on Table 1, the HEHR can be described as a collaborative record, whose guardian is a service acting on the behalf of the user, actors that interact with the patient or the patient can create data and the patient defines the sharing and access control. Hence the patient can choose the provider of the deposit service, detaching the storing cost of the health provider.

With such kind of record, it is expected that more information is deposited, gathering clinical information with other health related data. As an example, consider sport activity monitoring, athletic training programs, also other information produced by complementary medicine procedures. Moreover, new paradigms, such as home care, remote patient monitoring, and elderly care, can bring added value to the patient health record, as coping with the need of storing the information produced in such scenarios, through the collaboration paradigm. This contributes to the creation of the longitudinal patient-centric electronic health record.

\section{CONCLUSIONS}

This paper analyzed the two main streams of digital health records, the EHR and PHR, and how they cope with new challenges resulting from patient mobility and freedom of healthcare providers choice, along with the liberalization of healthcare provision and also the need of enabling records for future services and types of information.

It was present a Hybrid Electronic Health Record, that empowers the patient to control the access to his medical information, enables the easily access of new services from diverse providers to the record and maintains the integrity of the clinical information. Enabling that a longitudinal patient-centered electronic health record could be create by the collaboration of all the actors.

We are currently developing an framework 
that will enable such type of hybrid records, taking advantage of existing interfaces between EHR and PHR, namely a local EHR based upon OpenEHR and Google Health API. Further work is related to the implementation of a storage solution and also the evaluation data formats, namely the XML serialization from a CDA, CCR and OpenEHR archetypes.

\section{REFERENCES}

Chanda, R. (2002). Trade in health services. Bulletin of the World Health Organization, 80(2):158-63.

Costa, C., Freitas, F., Pereira, M., Silva, A., and Oliveira, J. (2009). Indexing and retrieving dicom data in disperse and unstructured archives. International Journal of Computer Assisted Radiology and Surgery, 4(1):71-77.

Dolin, R., Alschuler, L., Boyer, S., Beebe, C., Behlen, F., Biron, P., and Shabo Shvo, A. (2006). Hl7 clinical document architecture, release 2. Journal of the American Medical Informatics Association, 13(1):30.

Eysenbach, G. (2008). Medicine 2.0: social networking, collaboration, participation, apomediation, and openness. Journal of medical Internet research, 10(3):e22.

Ferranti, J., Musser, R., Kawamoto, K., and Hammond, W. (2006). The clinical document architecture and the continuity of care record. British Medical Journal, 13(3):245.

HIMSS (2010 (accessed July 19, 2010)a). Himss ehr definition. http://www.himss.org/ASP/ topics_ehr.asp.

HIMSS (2010 (accessed July 19, 2010)b). Himss phr definition. http://www.himss.org/ASP/ topics_phr.asp.

NCRR (2006). Electronic Health Records Overview. Technical report, MITRE.

OpenEHR (2007). Introducing openehr - revision 1.1. Technical report, OpenEHR.

Pedrosa, T., Lopes, R., Santos, J., Costa, C., and Oliveira, J. (2010). Towards an EHR architecture for mobile citizens. In HealthInf 2010 Proceedings, pages 288-293, Valencia, Spain. INSTICC-Institute for Systems and Technologies of Information, Control and Communication.

Santos, J., Pedrosa, T., Costa, C., and Oliveira, J. (2010). Modelling a portable personal health record. In HealthInf 2010 Proceedings.

Smith, K. and Kalra, D. (2008). Electronic health records in complementary and alternative medicine. International journal of medical informatics, 77(9):576-88.
The European Economic and Social Committee and the comittee of regions (2007). Final report on the implementation of the commission's action plan for skills and mobility $\operatorname{com}(2002) 72$ final. Technical report, Commission of the European Communities.

The Lancet (2008). Electronic health records. The Lancet, 371(9630):2058-2058. 